\title{
Reduction of Blocking Artifacts by Cepstral Filtering
}

\author{
by \\ Nam Ik Cho
}

\begin{abstract}
We propose a convolution model for the blocking artifacts in compressed images and videos, and a cepstral filtering algorithm for reducing the artifacts. Since each of the horizontal or vertical line of the compressed image has abrupt changes at almost every 8th samples, it can be modeled as a convolution of the original sequence with an excitation signal of periodicity 8. Comparison of the cepstrums of the compressed and original images shows that the multiples of 8th samples of the compressed signal's cepstrum usually have larger magnitudes than those of the original's, which supports the proposed model. The proposed filtering algorithm is to reduce the magnitudes of every 8th samples of the cepstrum, and disperse the reduced values to the neighboring samples. The algorithm is conducted in the cepstrum domain for reducing only the excitation signal which is the cause of blocking artifacts. Hence, the high frequency contents are not reduced by the postprocessing, whereas the conventional lowpass filtering blurs the image.
\end{abstract}

Keywords : blocking artifacts, cepstrum, cepstral filtering, compressed image

\footnotetext{
N. I. Cho is with the School of Electrical Engineering, Seoul National University, Seoul 151-742, KOREA.

Contact address : Prof. Nam Ik Cho

School of Electrical Engineering

Seoul National University

Shillim-Dong, Kwanak-Ku

Seoul 151-742, KOREA

Email : nicho@snu.ac.kr
} 


\section{List of Symbols}

$x[n] \quad$ A sequence of pixel values, usually a row or column of an image.

$h[n] \quad$ An excitation signal

$y[n]$ Convolution of $x[n]$ and $h[n]$, or a row (column) of a compressed image.

$c[n] \quad$ Cepstrum of $x[n]$.

$p[n] \quad$ Cepstrum of $h[n]$.

$s[n] \quad$ Cepstrum of $y[n]$.

$\lambda \quad$ control parameter for the strength of filtering.

Number of Pages : 15

Number of Tables : 0

Number of Figures : 8

Keywords : blocking artifacts, cepstrum, cepstral filtering, compressed image filtering, compressed image 


\title{
Reduction of Blocking Artifacts by Cepstral Filtering
}

by

\author{
Nam Ik Cho
}

\begin{abstract}
We propose a convolution model for the blocking artifacts in compressed images and videos, and a cepstral filtering algorithm for reducing the artifacts. Since each of the horizontal or vertical line of the compressed image has abrupt changes at almost every 8th samples, it can be modeled as a convolution of the original sequence with an excitation signal of periodicity 8. Comparison of the cepstrums of the compressed and original images shows that the multiples of 8th samples of the compressed signal's cepstrum usually have larger magnitudes than those of the original's, which supports the proposed model. The proposed filtering algorithm is to reduce the magnitudes of every 8th samples of the cepstrum, and disperse the reduced values to the neighboring samples. The algorithm is conducted in the cepstrum domain for reducing only the excitation signal which is the cause of blocking artifacts. Hence, the high frequency contents are not reduced by the postprocessing, whereas the conventional lowpass filtering blurs the image.
\end{abstract}

Keywords : blocking artifacts, cepstrum, cepstral filtering, compressed image

\section{Introduction}

Since the performance of discrete cosine transform (DCT) approaches that of the optimal transform for highly correlated signals, most of current image and video compression standards employ block DCT for the reduction of spatial redundancy [1]. However, the images compressed by block transforms have visually annoying artifacts along the block boundaries, which is known as blocking artifacts [2]. For reducing the blocking artifacts at the decoder end, many postprocessing algorithms have been proposed [3,4]. According to [3], postprocessing algorithms are derived from two different view points, i.e., image enhancement and image restoration. An example of image enhancement technique is the filtering of images around the block boundaries [5]. The constrained 
optimization and projection onto convex sets (POCS) are the methods based on image restoration $[2,6-10]$. Since the restoration algorithms usually take much computation time, the filtering technique is preferred for the real-time implementation $[5,11]$. For example, a simple deblocking filter is employed in the coding loop of the TMN for H.263+ [12,13]. All of the filtering methods in $[5,11,13]$ are based on the lowpass filters, and many of the constraints for the restoration algorithm are based on the smoothness constraints. As a result, the postprocessed images lose high frequency components inside the block boundaries, which blurs the images.

In this paper, in order to reduce the blocking artifacts while preserving the high frequency contents, we propose a cepstral filtering technique. We analyze the cepstrum of transform coded images and propose a convolution model for the blocking artifacts. Based on the convolution model, we also propose a cepstral filtering algorithm for reducing the cause of blocking artifacts. More precisely, since each of the horizontal or vertical line of the compressed image has abrupt changes at almost every 8th samples, corresponding samples of its cepstrum have larger magnitudes or changes than those of the cepstrum of the original signal. Based on this observation, it can be considered that the original signal is convolved with an excitation signal which has impulses at almost every 8th sample. For the reduction of blocking artifacts, we propose a cepstrum-domain filtering which reduces and disperses the components of excitation signal, i.e., the magnitudes of every 8th samples of the cepstrum. The experimental results show that the proposed ceptral filtering algorithm reduces the blocking artifacts while preserving the high frequency contents, whereas the conventional filtering blurs the images.

This paper is organized as follows. In section 2, we propose a convolution model for the blocking artifacts. In section 3, we analyze the cepstrum of a compressed image, and propose a cepstral filtering algorithm based on the convolution model. In section 4, experimental results and comparisons with a conventional method are presented. Section 5 provides concluding remarks.

\section{A Convolution Model for the Blocking Artifacts}

Fig. 1 shows the comparison of a row of original and compressed $512 \times 512$ Lena image. The Lena image is compressed by JPEG at $0.25 \mathrm{bpp}$, and the pixels at the positions from $(437,425)$ to $(437,472)$ are shown with those of the original image at the same positions. As observed from the Fig. 1, the compressed image loses its high frequency contents inside the block boundary. 
However, there are now new high frequency components due to abruptly changing pixel values at the block boundaries. In this paper, the compression process is considered as a combination of loss of high frequency details inside the block and a convolution process which generates the high frequency components at the boundaries. The estimation of lost high frequency components inside the block boundary is the scope of the image restoration methods. Hence, in this paper, we concentrate only on the convolution process and cepstral filtering along the block boundaries.

Fig. 1 Comparison of pixel values of original and compressed images.

Two kinds of blocking artifacts can be observed in Fig. 1, which can be roughly classified as shown in Fig. 2. The blocking artifacts observed around the pixel index 449 and 457 in Fig. 1 correspond to Fig. 2(a), and the ones around 433 and 465 to Fig. 2(b). In this section, we propose excitation signals and a convolution model which generate these typical blocking artifacts. Let $x[n]$ be a sequence of a row or a column of an image. Assuming that the $x[n]$ is a highly correlated signal, the convolution of $x[n]$ with the following signal $h[n]$ results in the signal of shape in Fig. 2(a).

$$
h[n]=\delta[n]+A_{1} \delta[n-8]+A_{2} \delta[n-16]+\cdots .
$$

The signal of shape in Fig. 2(b) can be generated by the following excitation signal

$$
h[n]=\delta[n]+\sum_{i=1}\left(-0.5 A_{i} \delta[n-8 i+1]+A_{i} \delta[n-8 i]-0.5 A_{i} \delta[n-8 i-1]\right) .
$$

As observed in Fig. 1, the actual excitation signal can be modeled as a combination of above signals. For example, a sequence $x[n]$ is convolved with an excitation signal $h[n]$ shown in Fig. 3(a) which is a random combination of eqs. (1) and (2). The input $x[n]$ and the convolution result $y[n]=h[n] * x[n]$ up to 64th samples are shown in Fig. 3(b), which has artifacts like the ones in Fig. 2.

Fig. 2 Typical shapes of scan lines at the block boundary.

Fig. 3 An example of the convolution of a sequence with an excitation signal. 


\section{Cepstrum Analysis of a Compressed Image and a Cepstral Filtering Algorithm}

In this section, we propose a deblocking algorithm based on the convolution model suggested above. Let $c[n], p[n]$ and $s[n]$ be the cepstrum of $x[n], h[n]$, and $y[n]$, respectively. In the computation of the cepstrum of each signal, it should be noted that $y[n]$ is the linear convolution of $x[n]$ and $h[n]$. Hence, for the computation of DFT of $x[n]$ and $h[n]$, zero padded sequences are used. More precisely, if $x[n]$ is a sequence of length $N$, then $L$ zeros are padded at the end of the sequence, where $L \geq N$ and $L+N=2^{m}$. Then $2^{m}$-point FFT and IFFT are performed for the computation of the cepstrums.

From the definition of cepstrum [15], the cepstrum sequences are related by

$$
s[n]=c[n]+p[n]
$$

As an example, Fig. 4 shows the cepstrums of the signals shown in Fig. 3, which verifies that $s[n]$ is the sum of $c[n]$ and $p[n]$. Since the length of the sequences in the example of Fig. 3 is 64 , the length of the cepstrum sequences is 128 as explained above, where only the sequences from 5 to 50 are shown in the Fig. 4. Given a compressed image, the cepstrum of its row or column is $s[n]$ according to our model. If we have exact knowledge about the excitation cepstrum $p[n]$, then the postprocessing is just to subtract $p[n]$ from $s[n]$. However, knowledge of $p[n]$ requires the original image, wchich is impractical. But, because the magnitude or gradient change of $s[n]$ at almost every 8th sample is larger than that of $c[n]$ as shown in Fig. 4, we can derive a practical deblocking algorithm by reducing the magnitude changes.

Fig. 4 Cepstrums of the excitation signal, input and output.

For deriving the algorithm, let us first compare the cepstrums of compressed and original $512 \times$ 512 Lena images, as an example. The 437th row of the image is selected for the comparison. Since the number of samples is 512, as many zeros are padded at the end of the sequence and part of the cepstrums of each sequence are compared in Fig. 5. The comparison shows that the compressed image's cepstrum has larger magnitude and/or larger change of gradient around the multiples of 8th samples. 
Fig. 5 Comparison of the cepstrums of 437th row of original and compressed Lena images.

From the proposed model and the above observation, we propose a deblocking algorithm which subtracts some proportional values from every 8th samples of the cepstrum, and disperse the subtracted values to the neighboring samples. More precisely, defining $\lambda$ as a parameter which determines the strength of the filtering, the proposed algorithm is to perform the following processes on the cepstrum $s[n]$ :

$$
\begin{aligned}
s[8 i-k] & :=s[8 i-k]-a_{k}(1-\lambda) s[8 i] \\
s[8 i] & :=\lambda s[8 i]
\end{aligned}
$$

for each $i=1,2, \ldots$, and for each $k=1, \ldots, 7$, where $0<\lambda<1.0$, and $\sum_{k=1}^{7} a_{k}=1.0$ for preserving the overall image brightness. Note that the eq. (4b) can be rewritten as

$$
s[8 i]:=s[8 i]-(1-\lambda) s[8 i] .
$$

which means that a proportional value of $s[8 i]$ is subtracted from itself, and it is dispersed to the neighboring samples by (4a). Hence, smaller $\lambda$ results in stronger filter. The values of $a_{k}$ are determined experimentally, which will be discussed in the next section. The overall algorithm can be summarized as follows for an example of $512 \times 512$ image, which is repeated for each row and column.

- Algorithm

1. Make a sequence $y[n]$ which consists of a row (or column) followed by 512 zeros.

2. Perform DFT of $y[n]$, and let the result be $Y[k]$.

3. Compute $\log Y[k]$.

4. Perform IDFT of $\log Y[k]$, and let the result be $s[n]$, which is the cepstrum of $y[n]$.

5. Perform the cepstral filtering in eqs. (4a) and (4b) for $i=1,2, \ldots \frac{1024}{8}-1$.

6. Perform the inverse processes of 4,3 and 2 for obtaining the filtered time-domain sequence. 


\section{Experimental Results}

For the comparison with the conventional spatial domain filtering [5], the algorithm is performed for the JPEG compressed Baboon and Lena images. Fig. 6 shows the results for the Baboon image. It can be observed that the proposed algorithm reduces the blocking artifacts, and that the image processed by the proposed algorithm has less blurring compared to the one processed by the conventional filtering. For another comparison, images around the the nose are shown in Fig. 7. It can be observed that the high frequency contents are preserved and the blocking artifacts are reduced, while the PSNR is the same as that of the conventional filtering. Fig. 8 shows the results for the Lena image, where the effect of the parameter $\lambda$ is also shown. Larger $\lambda$ reduces blocking artifacts more effectively, but it also raises other frequency contents. In all the experiments, the parameters in eq. (4a) are set as $a_{1}=0.7, a_{2}=0.2, a_{3}=0.1$, and the rest 0 . That is, the samples nearer to the 8th samples are given more weights, in order not to disperse the cepstrum too much. Too much reduction and dispersion yields distorted image which has too much high frequency components. The set of parameters stated above yields subjectively better results than the other sets we have tried. But there is no definite rule for the decision of the parameters, and more refined experiments and analysis are required for better choice of the parameters.

Fig. 6 Comparison for Baboon image.

Fig. 7 Comparison for Baboon image (around the nose).

Fig. 8 Comparison for Lena image.

\section{Conclusions}

In this paper, we have proposed a deblocking algorithm based on the cepstrum-domain filtering. First, it has been found that a row or column of a compressed image can be roughly modeled by a convolution of the original sequence with an excitation signal. Also, by examining the cepstrum of compressed images, it has been verified that the magnitudes or gradient changes of the cepstrum

at almost every 8th samples have larger magnitudes that those of the original signal. Hence, by proportional reduction and dispersion of every 8th samples of the cepstrum, it has been shown that the blocking artifacts can be reduced. Since the filtering is performed in the cepstrum domain 
for removing only the excitation signal that is the cause of blocking artifacts, it has less blurring compared to the conventional algorithm based on the lowpass filtering.

\section{References}

[1] N. Ahmed, T. Natarajan, and K. Rao, Discrete cosine transform, IEEE Trans. Computers, 23 (1), January 1974, pp. 90 - 93.

[2] Y. Yang, N. P. Galatsanos, and A. K. Katsaggelos, Regularized reconstruction to reduce blocking artifacts of block discrete cosine transform compressed images, IEEE Trans. Circuits and Systems for Video Technology, 3 (6), December 1993, pp. 421 - 432.

[3] M.-Y. Shen and C.-C. J. Kuo, Review of postprocessing techniques for compression artifact removal, Journal of Visual Communication and Image Representation, 9 (1), March. 1998, pp. 2 - 14.

[4] T. Meier, K. N. Ngan, and G. Crebbin, Reduction of blocking artifacts in image and video coding, IEEE Trans. Circuits and Systems for Video Technology, 9 (3), April 1999, pp. 490 500 .

[5] H. C. Reeves, and J. S. Lim, Reduction of blocking effects in image coding, Optical Engineering, 23, January/February 1984, pp. 34 - 37.

[6] Y. Yang, N. P. Galatsanos, Projection-based spatially adaptive reconstruction of blocktransform compressed images, IEEE Trans. Image Processing, 4 (7), December 1993, pp. $896-908$.

[7] H. Paek, R.-C. Kim, and S. U. Lee, On the POCS-based postprocessing technique to reduce the blocking artifacts in transform coded images, IEEE Trans. Circuits and Systems for Video Technology, 8 (3), June 1998, pp. 358 - 367.

[8] R. Rosenholtz and A. Zakhor, Iterative procedure for reduction of blocking effects in transform image coding, IEEE Trans. Circuits and Systems for Video Technology, 2 (1), March 1992, pp. $91-94$.

[9] M. Crouse, and K. Ramchandran, Nonlinear constrained least squares estimation to reduce artifacts in block transform-coded images, Proc. of ICIP 95, 1, 1995, pp. 462 - 465.

[10] T. K. Kim and J. K. Paek, Fast image restoration for reducing block artifacts based on adaptive constrained optimization, Journal of Visual Communication and Image Representation, 9, (3), September 1998, pp. 234 - 242.

[11] B. Ramarmurthi and A. Gersho, Nonlinear space variant postprocessing of block coded images, IEEE Trans. Acoust., Speech, Signal Processing, 34 (5), October 1986, pp. 1258 - 1267.

[12] ITU Telecom. Standardization Sector of ITU, Video codec test model near-term, Version 8 (TMN8), Release 0, H.268 Ad Hoc Group, June 1997. 
[13] G. Côté, B. Erol, M. Gallant, F. Kossentini, H.263+: Video Coding at Low Bit Rates, IEEE Circuits and Systems for Video Technology, 8 (7), November 1998, pp. 849 - 866.

[14] CCITT Rec. T.81, Information Technology - Digital Compression and Coding of ContinuousTone Still Images - Requirements and Guidelines, September. 1992.

[15] A. V. Oppenheim, and R. W. Schafer, Digital Signal Processing, Prentice-Hall, Englewood Cliffs, NJ, 1975, Chapter 10, pp. 490 - 500. 


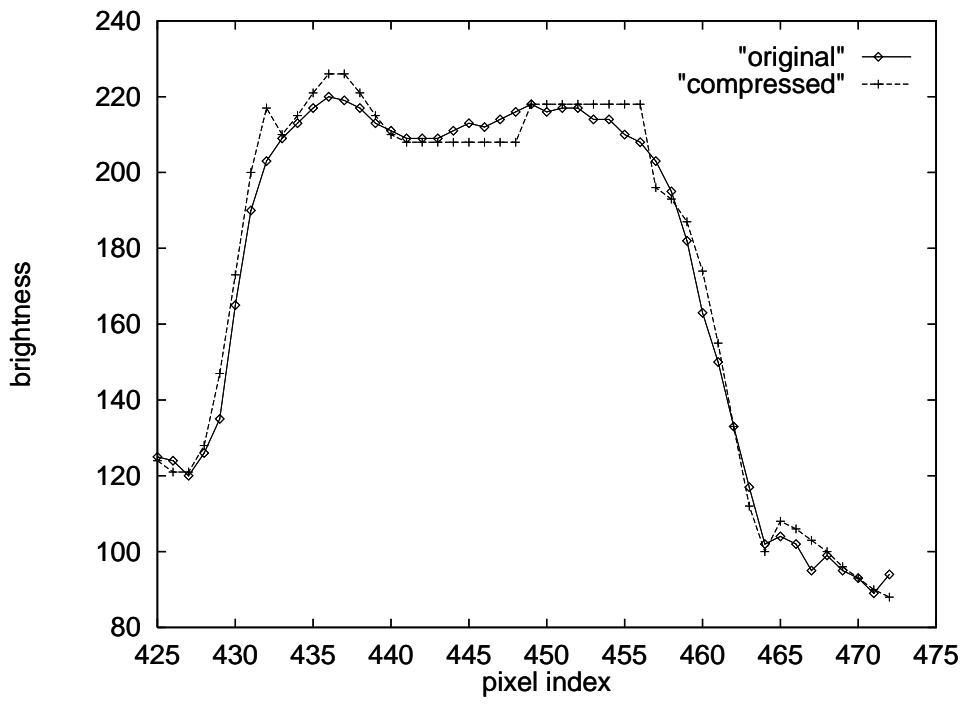

Figure 1: Comparison of pixel values of original and compressed images.

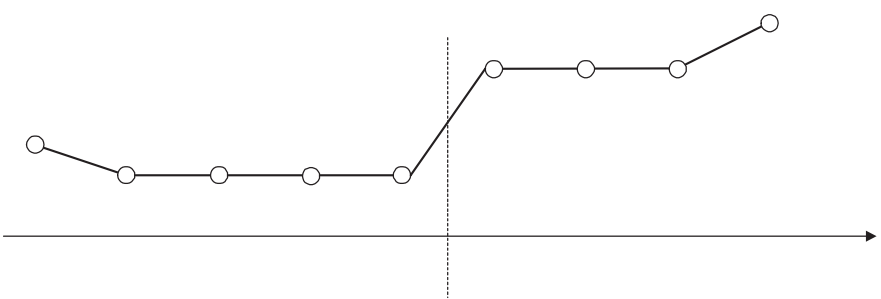

(a)

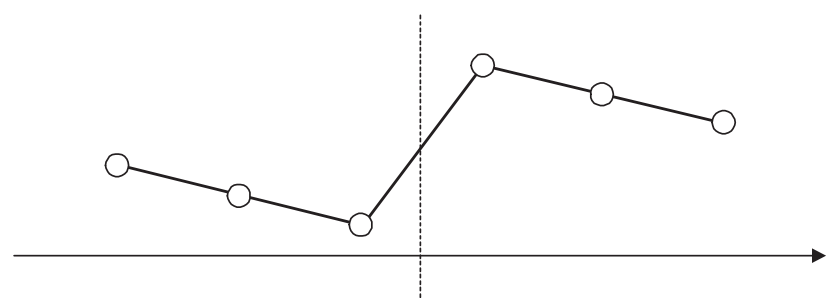

(b)

Figure 2: Typical shapes of scan lines at the block boundary. 


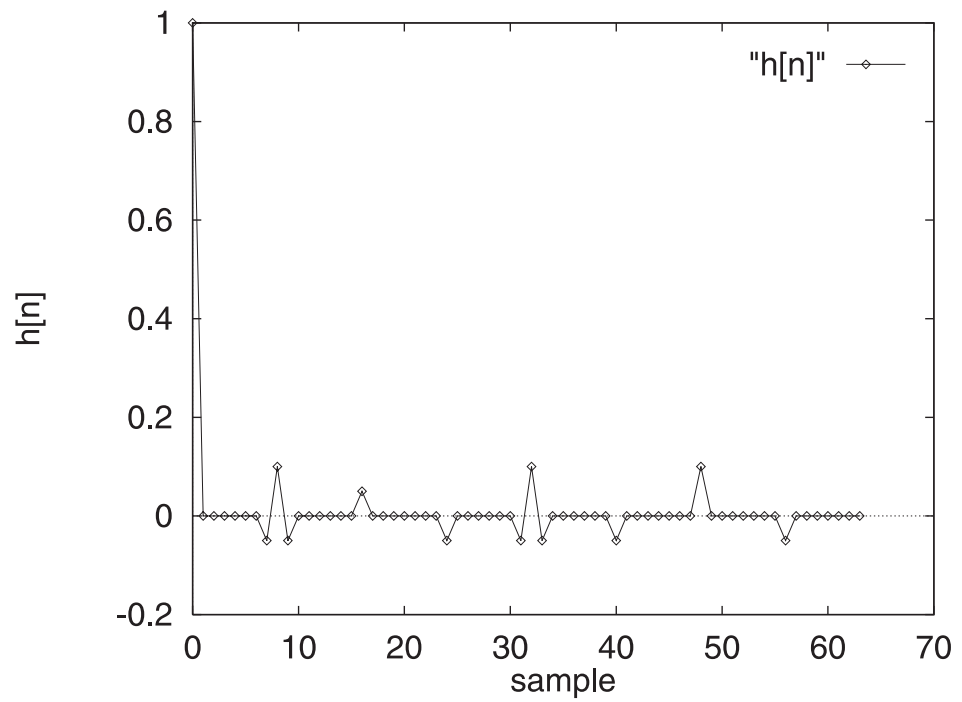

(a) excitation signal

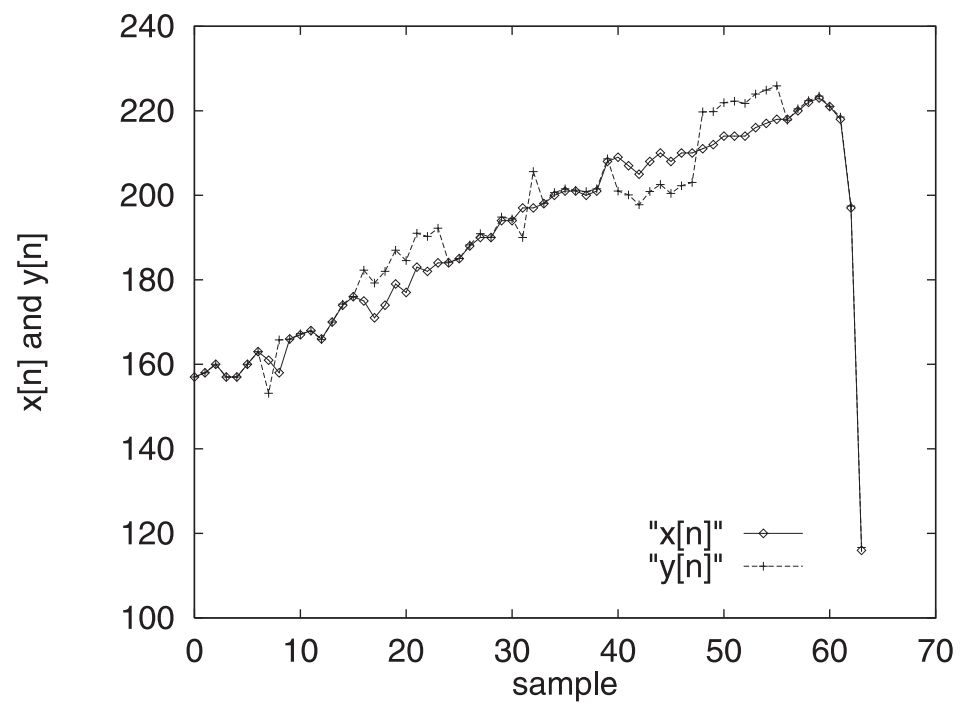

(b) input and output

Figure 3: An example of the convolution of a sequence with an excitation signal. 


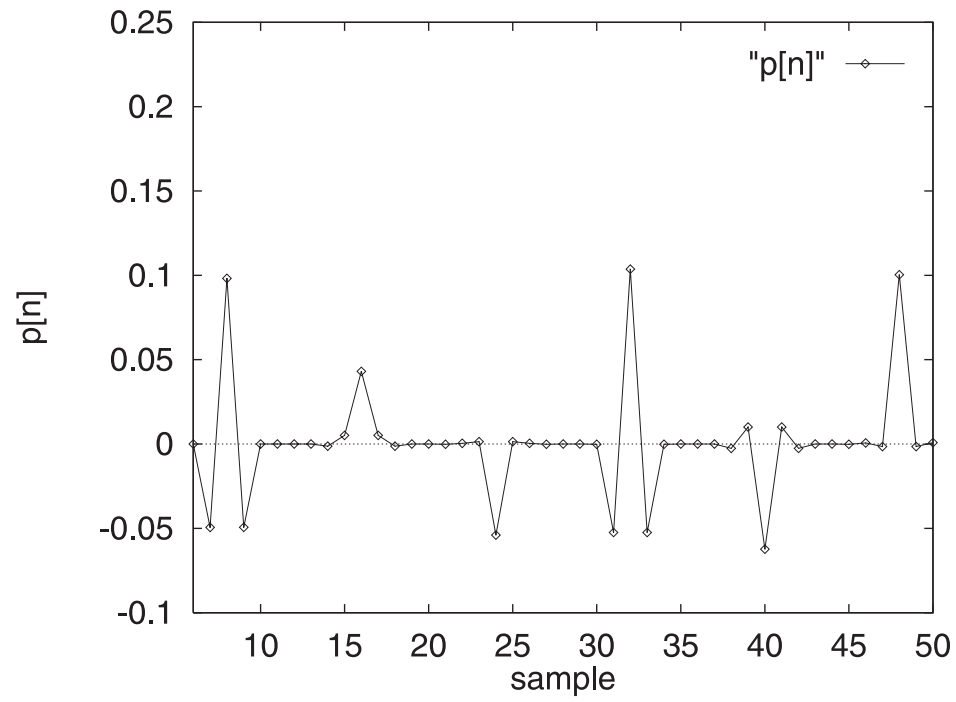

(a) $p[n]$ : cepstrum of excitation signal $h[n]$

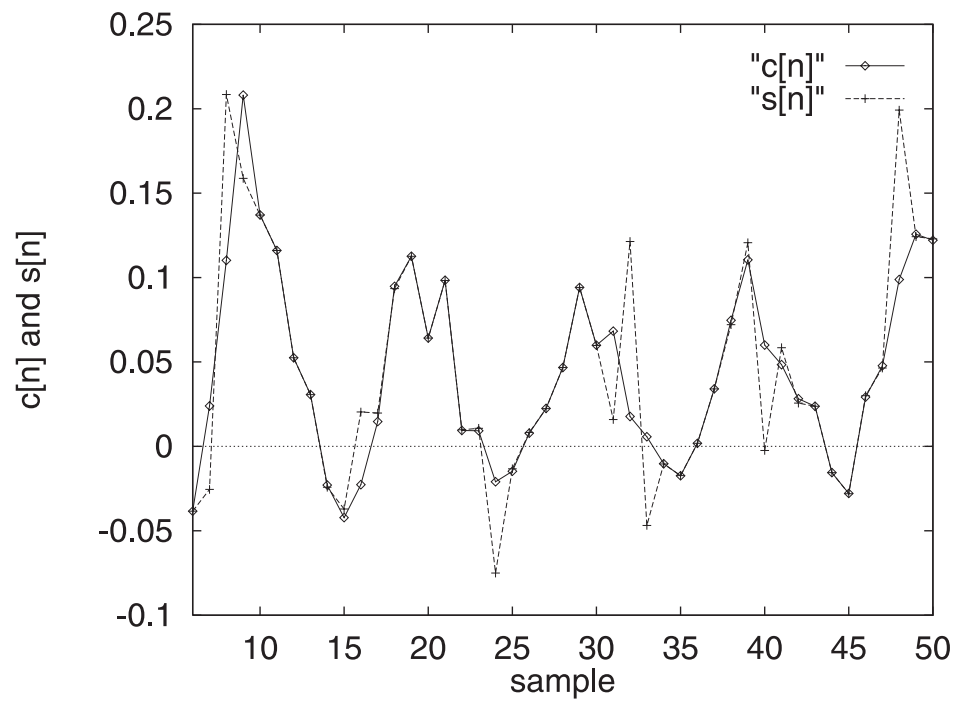

(b) $c[n]$ and $s[n]$ : cepstrums of input $x[n]$ and output $y[n]$, respectively.

Figure 4: Cepstrums of the excitation signal, input and output. 


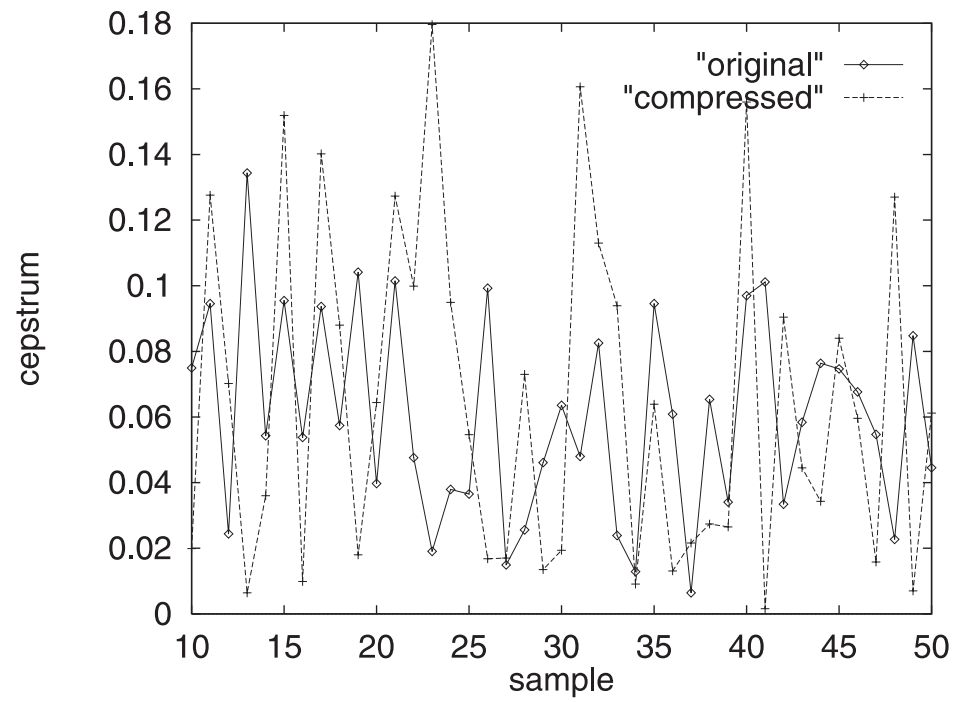

(a) Comparison of cepstrum samples from 10 to 50 .

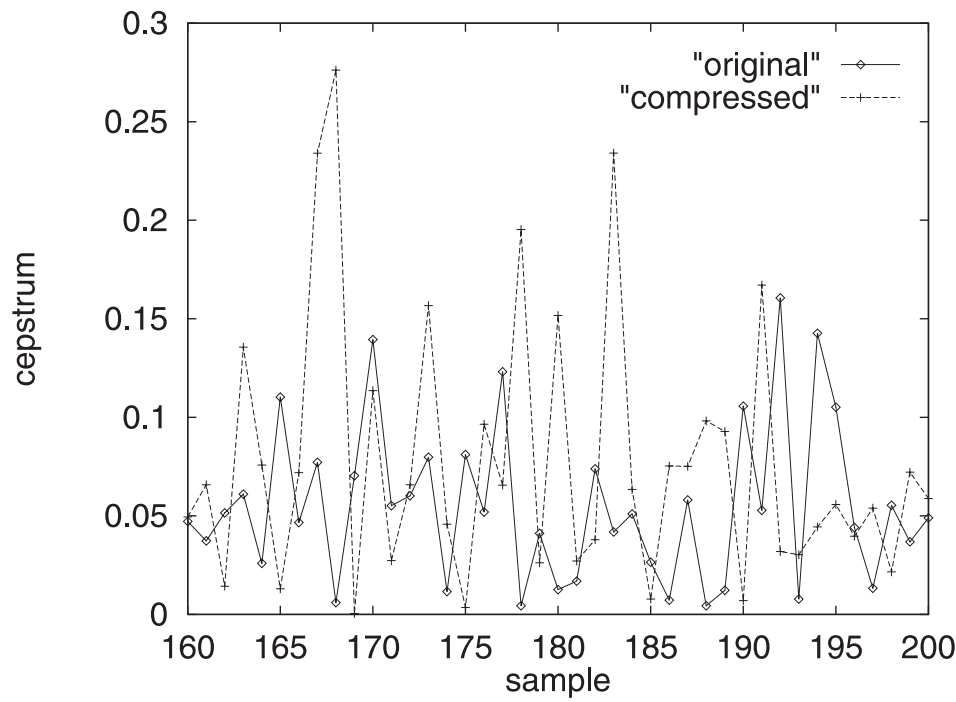

(b) Comparison of cepstrum samples from 160 to 200 .

Figure 5: Comparison of the cepstrums of 437th row of original and compressed Lena images. 


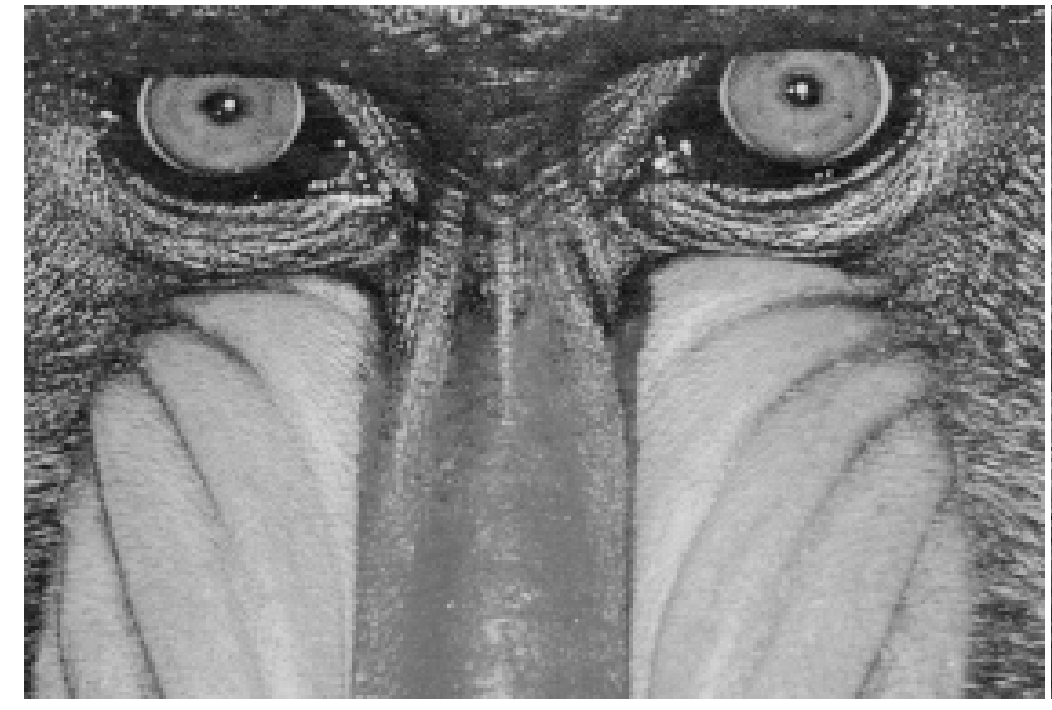

(a) original

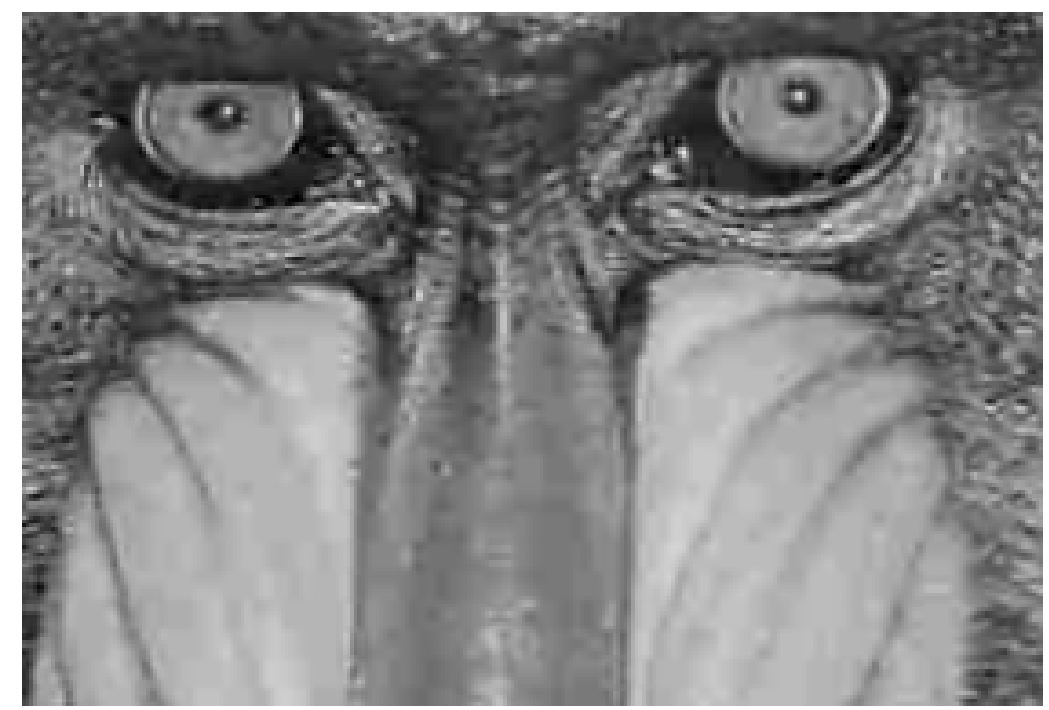

(c) conventional filtering [5] (24.2dB)

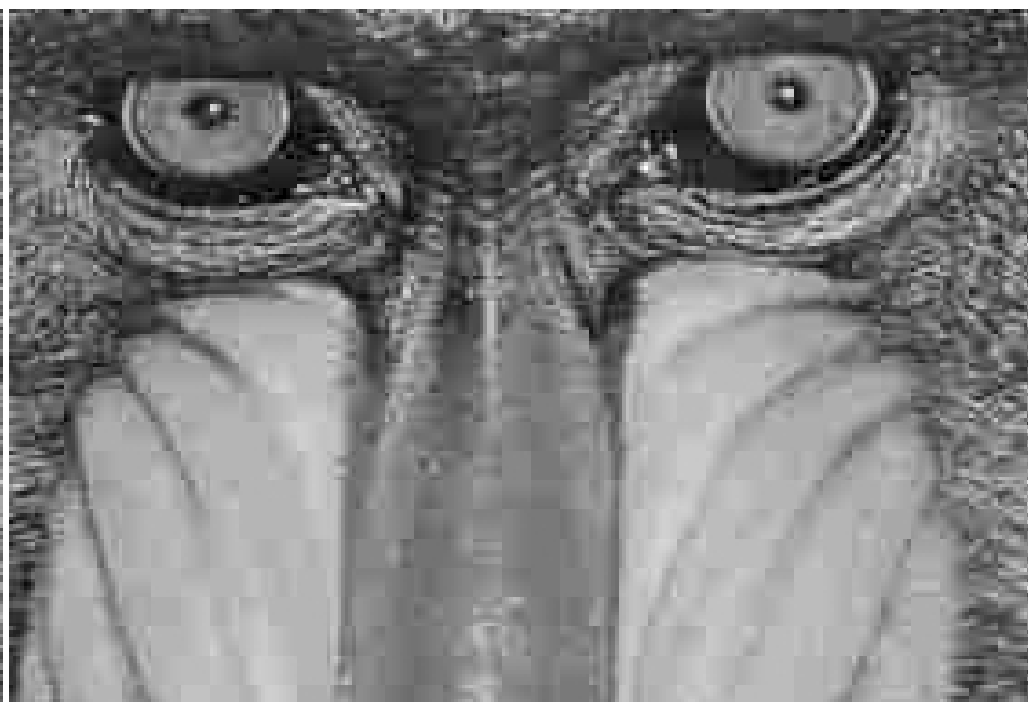

(b) JPEG (24.5dB)

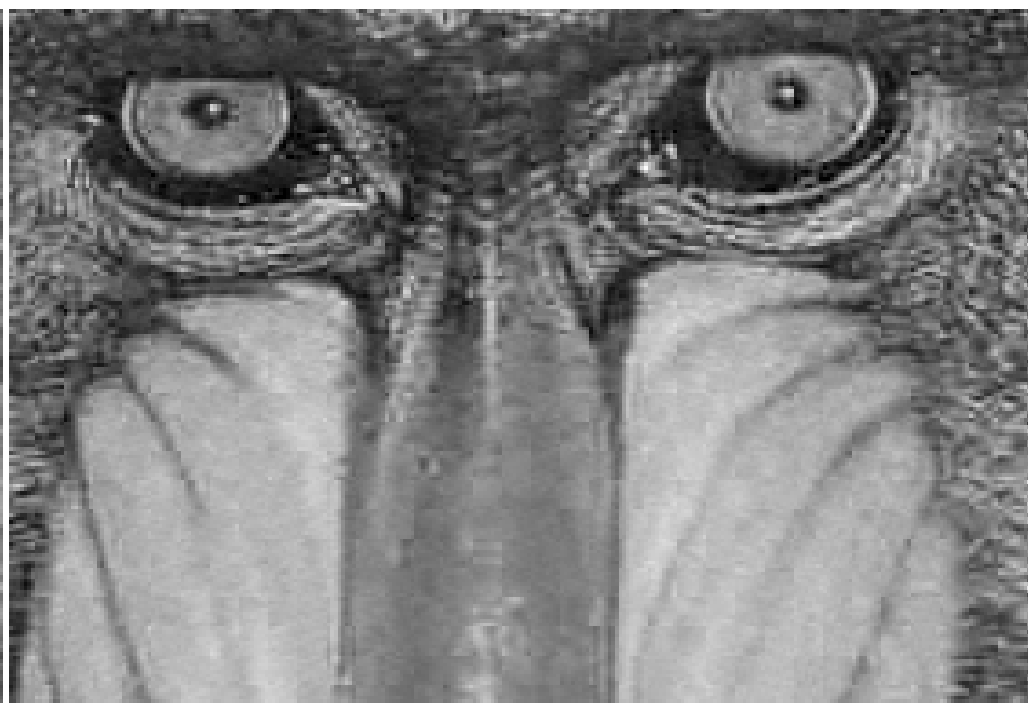

(d) proposed (24.2dB, $\lambda=0.7)$

Figure 6: Comparison for Baboon image 


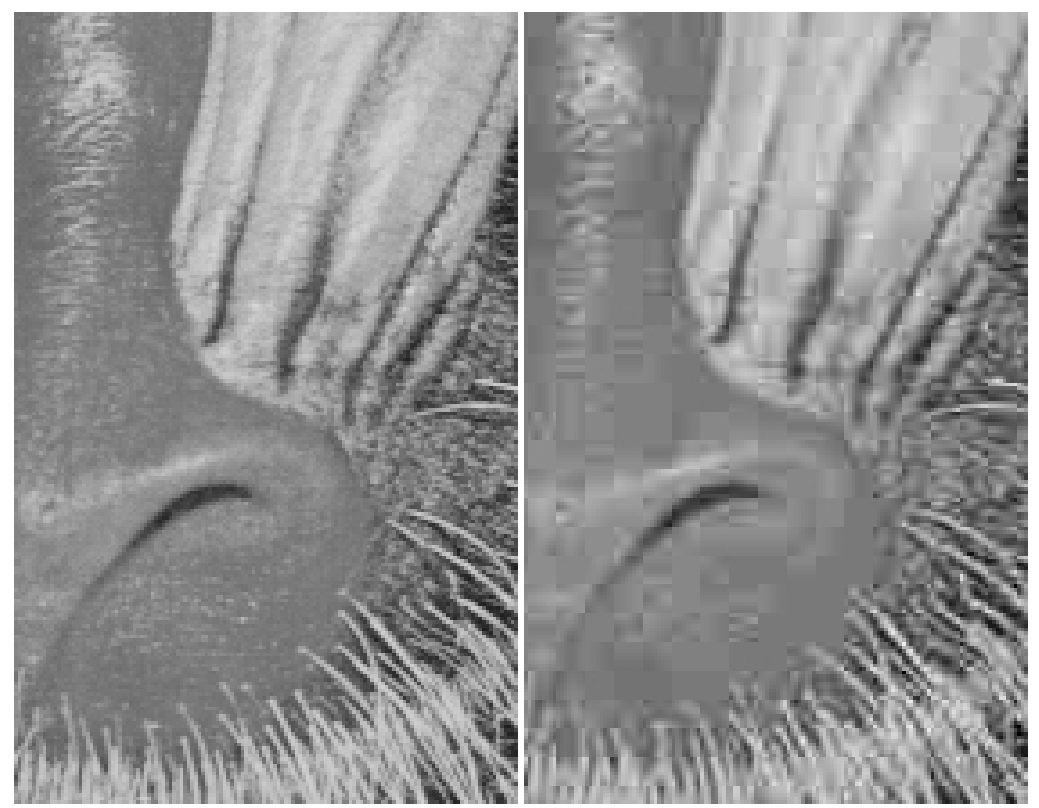

(a) original

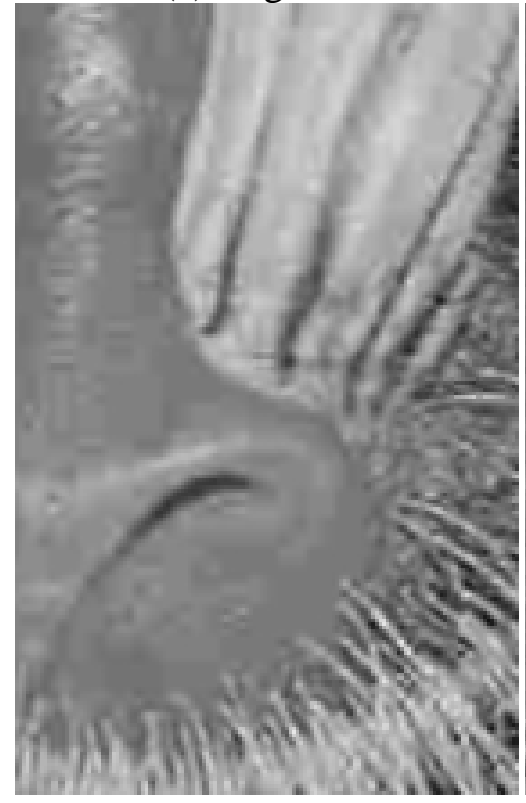

(c) filtering (b) JPEG

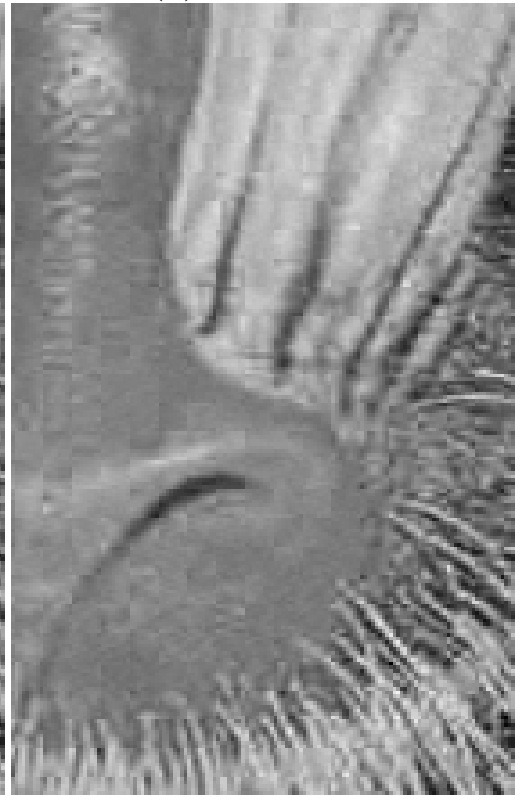

(d) proposed

Figure 7: Comparison for Baboon image (around the nose). 


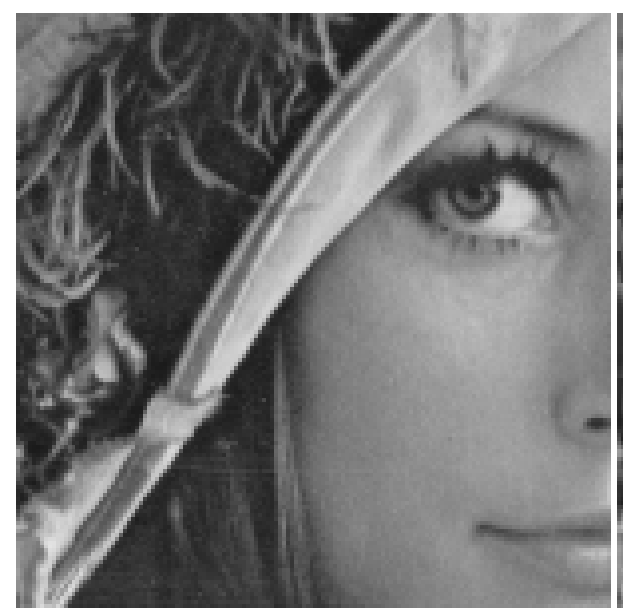

(a) original

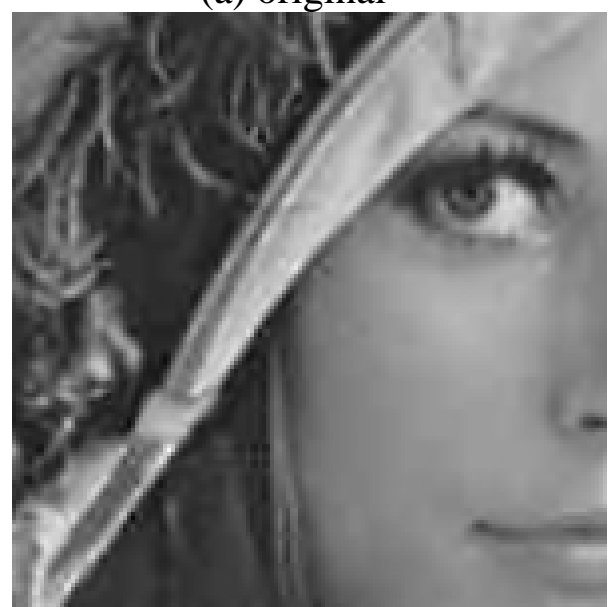

(c) filtering $(33.7 \mathrm{~dB})$

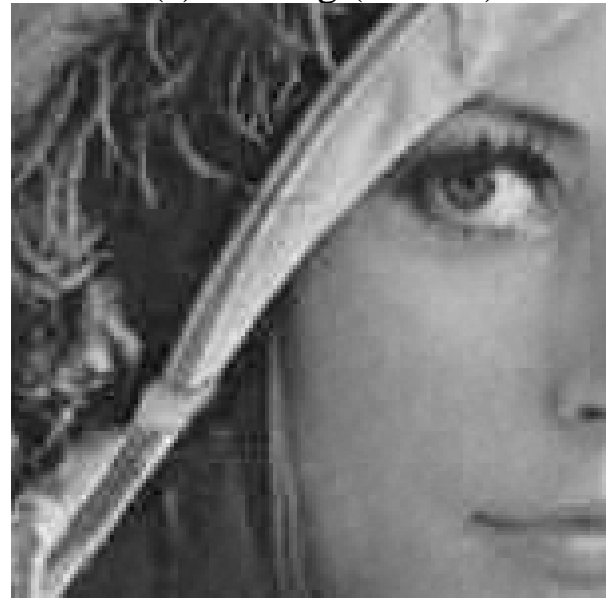

(e) proposed $(\lambda=0.8,33.3 \mathrm{~dB})$

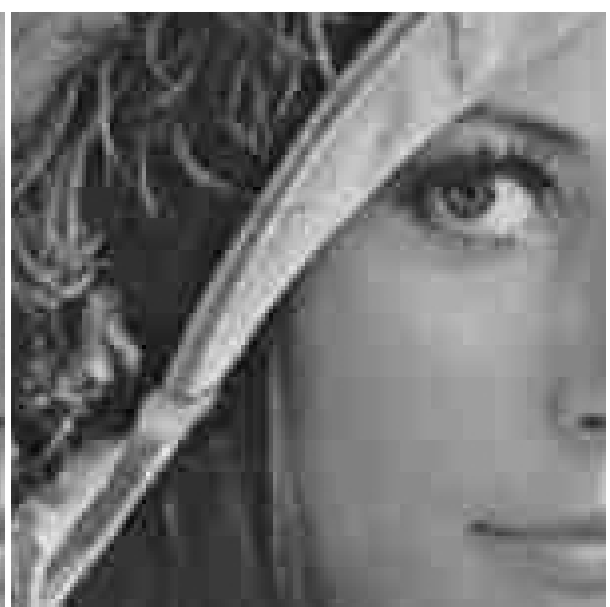

(b) JPEG (33.6dB)

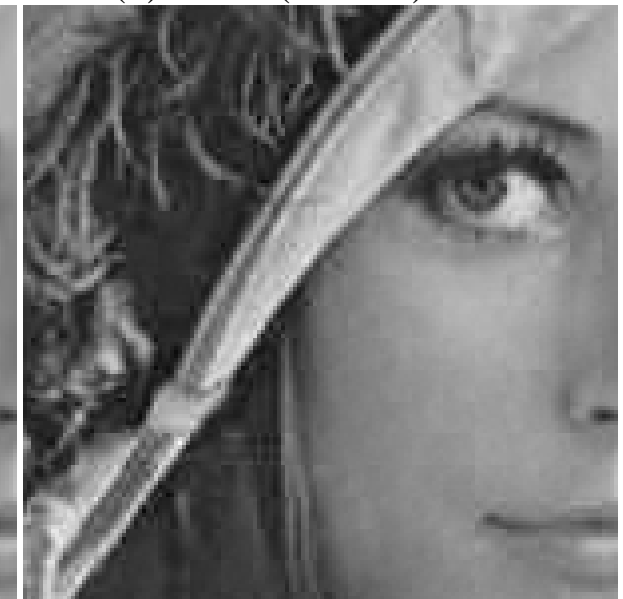

(d) proposed $(\lambda=0.85,33.5 \mathrm{~dB})$

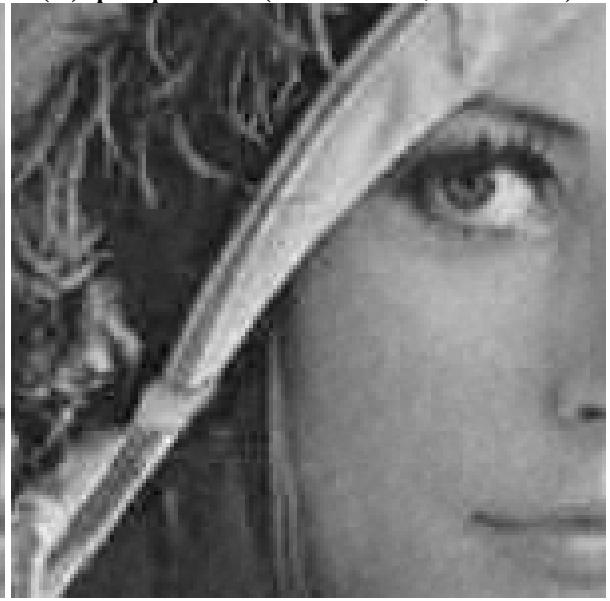

(f) $\operatorname{proposed}(\lambda=0.7,32.7 \mathrm{~dB})$

Figure 8: Comparison for Lena image. 